\title{
Performance of Wrist Photoplethysmography in Monitoring Atrial Fibrillation in Post Cardiac Surgery Patients
}

\author{
Adrian Tarniceriu ${ }^{1}$, Vilma Vuohelainen ${ }^{2}$, Serj Haddad ${ }^{1}$, Tuomas Halkola ${ }^{3}$, Jakub Parak ${ }^{3}$, Jari \\ Laurikka $^{2,4}$, Antti Vehkaoja ${ }^{3,4}$ \\ ${ }^{1}$ PulseOn SA, Neuchatel, Switzerland \\ ${ }^{2}$ Department of Cardiothoracic Surgery, Heart Center, Tampere University Hospital, Finland \\ ${ }^{3}$ PulseOn Oy, Espoo, Finland \\ ${ }^{4}$ Faculty of Medicine and Health Technology, Tampere University, Tampere, Finland
}

\begin{abstract}
New methods for unobtrusive long-term monitoring of arrhythmias are needed. We evaluated the accuracy of wrist-worn optical heart rate monitor in beat-to-beat interval estimation and performance in classifying the rhythm in sinus or atrial fibrillation with 30 post cardiac surgery patients $(69.3 \pm 6.9$ years $)$ in 24 -hour recordings. The recordings were made during the first post-operative days when the patients are still relatively stationary and are in high risk of getting post-surgery atrial fibrillation.

The results show 10.04 ms mean absolute error in beatto-beat intervals. Atrial fibrillation was analysed in 5minute segments. $100 \%$ sensitivity and $96.1 \%$ specificity were obtained. The specificity was increased to $99.74 \%$ when the data segments with large number of ventricular and supra ventricular extra systoles were removed. The results show that the performance of optical heart rate monitoring technology is adequate for screening of atrial fibrillation at least in stationary conditions.
\end{abstract}

\section{Introduction}

Atrial fibrillation (AF) is the most common cardiac arrhythmia. It is associated with increased risks (up to five fold) of thromboembolic ischemic stroke, hypertension, heart failure, and myocardial infarction [1- 3]. The prevalence of $\mathrm{AF}$ increases strongly with age. According to Zoni-Berisso et al., the prevalence is $0.12 \%-0.16 \%$ in people less than 49 years, $3.7 \%-4.2 \%$ in people between $60-70$ years, and $10 \%-17 \%$ in people aged 80 years and older [3]. The exact numbers naturally vary between studies. Common symptoms of AF include general fatigue, dizziness, shortness of breath, rapid heartbeats, and fluttering feeling in the chest, but $\mathrm{AF}$ may also be asymptomatic. Especially identifying the patients with subclinical silent AF is a challenge [4]. It is estimated that in approximately one third of the cases, atrial fibrillation occurs as asymptomatic, silent $\mathrm{AF}[5,6]$ but the exact numbers are difficult to obtain because silent AF is usually found by accident during some other medical examination.

European Society of Cardiology recommends systematic screening programs for people older than 75 years or belonging to a group of high risk of stroke, and opportunistic screening for those older than 65 years [7]. Screening programs actually being executed are, however, very limited, Belgium being one of the most progressive countries [8].

Current methods for detection and monitoring of atrial fibrillation include continuous ECG monitoring with multi lead Holter or single lead patch device [9], intermittent measurement with a device held between the hands [10] or pressed to the chest, and implanted loop recorder [11]. Being an implantable device, loop recorder is relatively expensive and thus in the past has been mainly used in diagnosing syncope [12]. On the other hand, the effectiveness of Holter monitoring is limited due to limited monitoring time. Intermittent measurement devices enable long monitoring time but their challenge is the detection of short paroxysmal and especially asymptomatic AF episodes. Therefore, alternative unobtrusive methods for screening and monitoring of AF are desired.

We evaluated the performance of a wrist-worn photoplethysmografic (PPG) device in monitoring cardiac rhythm and detecting AF in post-cardiac surgery patients in 24-hour recordings. While being comfortable to wear, wrist PPG could provide a solution for continuous $24 / 7$ monitoring of cardiac rhythm. 


\section{Materials and Methods}

\subsection{Data Collection}

30 cardiac surgery patients (9 female, 21 male, $69.3 \pm$ 6.9 years old) were recruited for the study from Cardiac surgery ward at Tampere University Hospital. The majority of the subjects had undergone either coronary artery bypass or mitral valve replacement surgery. Some had undergone a combination surgery of the two. This group was chosen due to their high risk of post-operative atrial fibrillation [13]. Subjects were monitored for 24 hours with a wrist-worn PPG monitor (PulseOn Oy, Espoo, Finland) shown in Figure 1, leading to roughly 697 hours of data. 5-lead Holter ECG device, eMotion Faros 360 (Bittium Biosignals Oy, Kuopio, Finland) was used as the reference. The monitoring was started on $2^{\text {nd }}$ to $4^{\text {th }}$ postoperative day. The subjects were mostly staying in bed during the monitoring. A favorable statement was obtained for the study from the local ethical committee of Tampere University Hospital (R17038). All subjects gave their informed consents in writing.

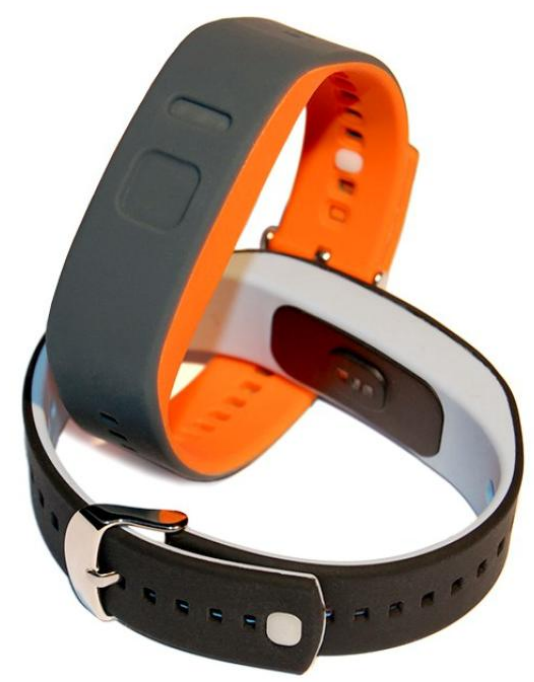

Figure 1. PulseOn optical wrist-worn heart rate monitor used in the study.

\subsection{Arrhythmia Detection}

The PulseOn wrist device uses proprietary algorithms of the company for detecting heartbeats, calculating interbeat-intervals (IBI), and performing the arrhythmia detection. The algorithms are designed in such way that they can all be embedded into the device and run in real time. In this study, however, the signal analysis was performed off-line with Matlab. A simplified block diagram of the algorithm is shown in Figure 2.

The first step is to detect the heartbeats and estimate the
IBIs based on the PPG signal. Each IBI is also assigned with a label providing signal quality or heartbeat reliability information. This information is used to discard unreliable beats from further processing. This data is then further used to detect AF in beat-to-beat basis (Arrhythmia detection block in Fig. 2). The method performs the analysis based on approximately 30 seconds of data assigning each heartbeat a label, whether it is sinus rhythm, arrhythmia (Arr), or undetermined. The method allows up to half of the heartbeats or inter-beat intervals being marked as "unreliable" still being able to make a decision. Single ectopic beats such as ventricular extra systoles are found based on the morphology of the IBI tachogram signal and marked before the arrhythmia detection step. The method has been described in more detail in [14] and the IBI estimation accuracy in $[15,16]$. Ectopic beat detection method is described in more detail in [17].

The final rhythm classification is made in 5-minute segments. If the segment contains at least a certain amount of IBIs marked as Arr in the first phase, the whole segment is marked as arrhythmia. Otherwise, if the segment contains at least the same amount of IBIs marked as SR, the whole segment is marked as SR. Otherwise, e.g. if the segment contains too many "Undetermined" IBIs, the whole segment is marked as "Undetermined". The method can detect arrhythmia episodes as short as 30 IBI, while being resistant to isolated IBI classification errors.

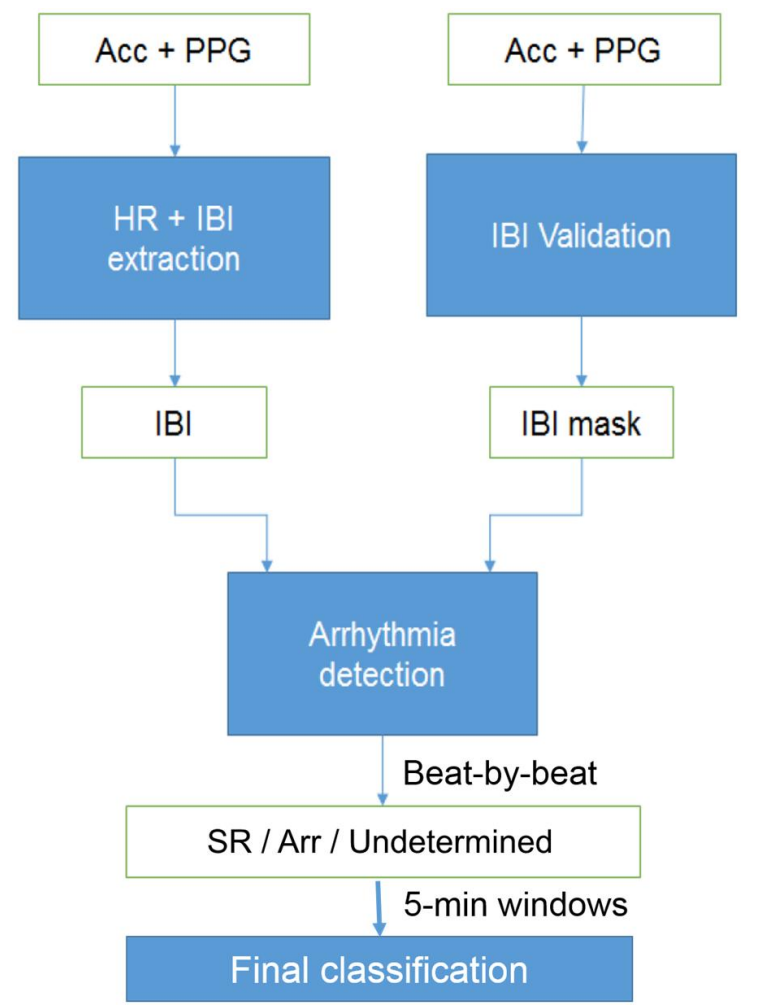

Figure 2. Data flow block diagram of PulseOn arrhythmia analysis method. 


\section{Results and Discussion}

$12.3 \%$ of the 5 -minute segments were discarded due to inadequate signal quality and the remaining $87.7 \%$ of the data was classified to arrhythmia or sinus rhythm. Three out of the 30 subjects had actual AF during the monitoring period leading to 22 hours of AF data. All data segments during AF were correctly labeled as arrhythmia providing $100 \%$ sensitivity for AF. From the non-AF data, 96.10\% was classified as sinus rhythm. Most of the arrhythmia classification caused by other than AF, resulted from the presence of very frequent ventricular and supraventricular ectopic beats ( $>10$ per minute) that had not been removed by the ectopic beat detector of the algorithm, mostly due to frequency of their occurrence. Altogether eight subjects had such episodes. In four of them, the episodes lasted altogether more than one hour (in one case $>9$ hours, i.e. 1135 -minute segments). If these segments are ignored, i.e. only sinus rhythm data is observed, the false positive rate becomes $0.26 \%$, i.e. the specificity becomes $99.74 \%$.

We also evaluated the accuracy of the initial inter-beatinterval estimation by comparing IBIs provided by the wrist device with the R-R-intervals estimated from the ECG signal. The mean absolute error in estimating the individual IBIs was found to be $10.04 \mathrm{~ms}$. Table 1 summarizes the results of the study.

Table 1. The performance of IBI estimation and AF detection.

\begin{tabular}{ll}
\hline Parameter & Value \\
\hline Classified data & $87.7 \%$ \\
IBI MAE & $10.04 \mathrm{~ms}$ \\
AF sensitivity & $100 \%$ \\
AF specificity & $96.10 \%$ \\
AF specificity & $99.74 \%$ \\
(when ignoring data containing excess \\
amount of ectopic beats) \\
\hline
\end{tabular}

Fig. 3 shows an example of inter-beat interval detection in addition to mask provided by the signal quality estimation algorithm. As can be seen, the SQE sometimes also marks the correct IBIs as unreliable. This tradeoff, however, has to be accepted in order to achieve good IBI accuracy and specificity in AF detection.

\section{Conclusion}

The performance of wrist-worn optical heart rate monitoring in atrial fibrillation detection in stationary conditions was demonstrated. The method achieved $100 \%$ sensitivity but the large number of frequent ectopic beats caused arrhythmia notifications in the device algorithm, which decreases the specificity if using the device specifically for atrial fibrillation detection.
Improving the performance of the method of handling the ectopic beats and adding this as an additional class in rhythm classification will be an important part of our future work.

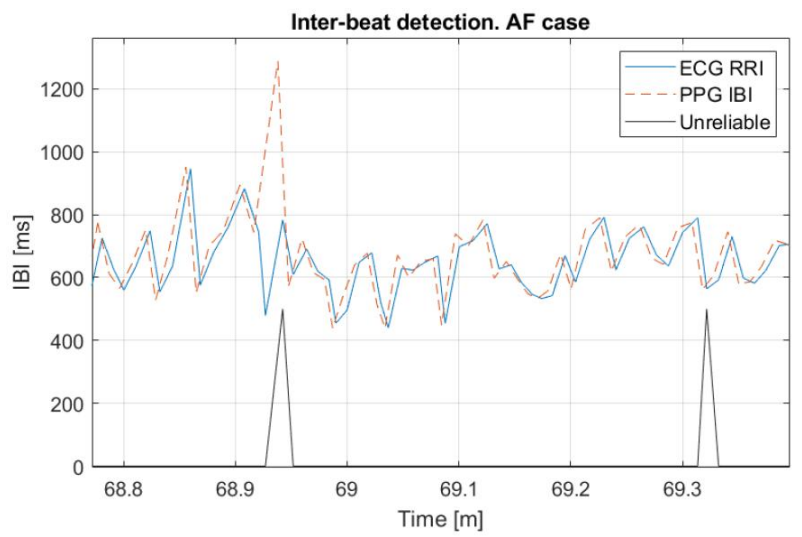

Figure 3. Example of IBI comparison to ECG RRI data. Black arrows show the individual IBIs marked as unreliable.

\section{Acknowledgments}

We would like acknowledge the research nurses of Heart Hospital of Tampere University Hospital for performing the data collection.

\section{References}

[1] R A. Vermond, B. Geelhoed, N. Verweij, R. G. Tieleman, P. van der Harst, H. L. Hillege, W. H. van Gilst, I. C. van Gelder, M. and Rienstra, "Incidence of atrial fibrillation and relationship with cardiovascular events, heart failure, and mortality," J. Am. Coll. Cardiol. vol. 66, no. 9, pp.10001007, Sep. 2015.

[2] V. Ruddox, I. Sandven, J. Munkhaugen, J. Skattebu, T. Edvardsen, and J. E. Otterstad, "Atrial fibrillation and the risk for myocardial infarction, all-cause mortality and heart failure: A systematic review and meta-analysis," Eur J Prev Cardiol, vol. 24, no. 14, 1555-1566, Sep. 2017.

[3] M. Zoni-Berisso, F. Lercari, T. Carazza, and S. Domenicucci, "Epidemiology of atrial fibrillation: European perspective," Clin Epidemiol, vol. 6, pp. 213-220, Jun 2014.

[4] T. Lewalter and G. Boriani, "Relevance of Monitoring Atrial Fibrillation in Clinical Practice," Arrhythm Electrophysiol Rev, vol 1, no. 1, pp. 54-58, Sep 2012.

[5] P. E. Dilaveris and H. L. Kennedy, "Silent atrial fibrillation: epidemiology, diagnosis, and clinical impact," Clin Cardiol, vol. 40, no. 6, pp. 413-418, Jun 2017.

[6] A.J. Camm, G. Corbucci, and L. Padeletti, "Usefulness of continuous electrocardiographic monitoring for atrial fibrillation," Am J Cardiol, vol 110, no. 2, pp.270-276, Jul 2012.

[7] P. Kirchhof, et al. "2016 ESC Guidelines for the management of atrial fibrillation developed in collaboration with EACTS," Eur J Cardiothorac Surg, vol. 50, no.5, pp. e1-e88, Nov 2016. 
[8] M. Proietti, G. H. Mairesse, P. Goethals, C. Scavee, J. Vijgen, I. B lankoff,, Y. Vandekerckhove, and G. Y. Lip, “A population screening programme for atrial fibrillation: a report from the Belgian Heart Rhythm Week screening programme," Europace, vol. 18, no. 12, pp. 1779-1786, Dec 2016.

[9] S. R. Steinhubl, J. Waalen, A. M. Edwards, L. M. Ariniello, R. R. Mehta, G. S. Ebner, C. Carter, K. Baca-Motes, E. Felicione, T. Sarich, and E. J. Topol, "Effect of a homebased wearable continuous ECG monitoring patch on detection of undiagnosed atrial fibrillation: the mSToPS randomized clinical trial," Jama, vol. 320, no. 2, pp. 146155, Jul 2018.

[10] M. Stridh and M. Rosenqvist, "Automatic screening of atrial fibrillation in thumb-ECG recordings," In 2012 Computing in Cardiology, pp. 193-196, Sep 2012. IEEE.

[11]A. Bansal and J. Rajnish, "Portable out-of-hospital electrocardiography: A review of current technologies," $J$ Arrhythm, vol. 34, no. 2, pp. 129-138, Feb 2018.

[12] R. Sakhi, D. A. Theuns, R. E. Bhagwandien, M. Michels, A. F. Schinkel, T. Szili-Torok, F. Zijlstra, J. W. RoosHesselink, and S. C. Yap, "Value of implantable loop recorders in patients with structural or electrical heart disease," J Interv Card Electrophysiol, vol. 52, no. 2, pp. 203-208, Mar 2018.

[13] S. Helgadottir, M. I. Sigurdsson, I. L. Ingvarsdottir, D. O. Arnar, and T. Gudbjartsson, "Atrial fibrillation following cardiac surgery: risk analysis and long-term survival," $J$ Cardiothorac Surg, vol. 7, Sep 2012.
[14] A. Tarniceriu, J. Harju, Z. R. Yousefi, A. Vehkaoja, J. Parak, A. Yli-Hankala, and I. Korhonen, "The accuracy of atrial fibrillation detection from wrist photoplethysmography. a study on post-operative patients," In 2018 40th Ann Int Conf of the IEEE Eng Med Biol Soc (EMBC), Jul 2018, IEEE.

[15] J. Harju, A. Tarniceriu, J. Parak, A. Vehkaoja, A. YliHankala, and I. Korhonen, "Monitoring of heart rate and inter-beat intervals with wrist plethysmography in patients with atrial fibrillation," Physiol Meas, vol. 39, no. 6, 065007, Jun 2018.

[16] A. Tarniceriu, J. Harju, A. Vehkaoja, J. Parak, R. DelgadoGonzalo, P. Renevey, A. Yli-Hankala, and I. Korhonen, "Detection of beat-to-beat intervals from wrist photoplethysmography in patients with sinus rhythm and atrial fibrillation after surgery," In 2018 IEEE EMBS Int Conf Biomed \& Health Inform (BHI), pp. 133-136, Mar 2018, IEEE.

[17] S. Haddad, J. Harju, A. Tarniceriu, T. Halkola, J. Parak, I. Korhonen, A. Yli-Hankala, and A. Vehkaoja, "Ectopic Beat Detection from Wrist Optical Signals for Sinus Rhythm and Atrial Fibrillation Subjects," In Medit Conf Med Biol Eng Comp, pp. 150-158, Sep 2019, Springer.

Address for correspondence:

Antti Vehkaoja

Korkeakoulunkatu 3, 33720, Tampere, Finland. antti.vehkaoja@tuni.fi 